\title{
Cyanine-based colorimetric and fluorescent probe for the selective detection of diethylstilbestrol in seawater, shrimp and fish samples
}

\author{
Kun Yin ${ }^{\mathrm{a}, \mathrm{b}}$, Fabiao $\mathrm{Yu}^{\mathrm{a}}$, Dongyan Liu ${ }^{\mathrm{a}}$, Zhihong Xie ${ }^{\mathrm{a}}$, Lingxin Chen ${ }^{\mathrm{a}, *}$ \\ ${ }^{a}$ Key Laboratory of Coastal Environmental Processes and Ecological Remediation, Yantai Institute of Coastal Zone Research, Chinese Academy of Sciences, \\ Yantai 264003, China \\ ${ }^{\mathrm{b}}$ University of Chinese Academy of Sciences, Beijing 100049, China
}

\section{A R T I C L E I N F O}

\section{Article history:}

Received 16 July 2015

Received in revised form

30 September 2015

Accepted 6 October 2015

Available online 8 October 2015

\section{Keywords:}

Diethylstilbestrol

Fluorescent probe

Colorimetric analysis

Near-infrared

Electrostatic interaction

\begin{abstract}
A B S T R A C T
The synthetic estrogen drug diethylstilbestrol (DES) plays important roles in the treatment of estrogen deficiency disorders for human beings. The excessive intakes of DES can lead to physiological dysfunction and raise the risk of ovarian cancer, breast cancer and other diseases. However, it is still abused for improving the fat deposition or sex reversal procedure in aquatic economic creatures for pursuit of huge interests. DES can exist in aquatic products or polluted aquacultural seawater, which seriously threatens human health. Therefore, it is desirable to establish simple and sensitive methods for the detection of DES. In this work, we have developed a new colorimetric and fluorescent probe Cy-DES for the detection of DES with high sensitivity and selectivity. As a near-infrared probe, Cy-DES is able to avoid autofluorescence of dissolved organic compounds and maximize signal-to-background contrast. Taking advantage of the strong electrostatic interaction between the probe Cy-DES and DES, the spectroscopic properties of probe Cy-DES can be obviously changed in presence of DES. Under testing conditions, there is an excellent linearity within the range of $1-8 \mu \mathrm{M}(r=0.9997)$ and the detection limit is $0.2 \mu \mathrm{M}$. The probe Cy-DES is successfully applied for the detection of DES in spiked seawater, shrimp and fish samples. Additionally, the detection of DES can be directly achieved by naked eyes with the utilizing of probe Cy-DES. The developed method is of great potential for application in the on-site detection of DES.
\end{abstract}

(C) 2015 Elsevier B.V. All rights reserved.

\section{Introduction}

As one of the synthetic estrogen drugs, diethylstilbestrol (DES) is usually employed to the prevention and treatment of pregnancy complications [1]. The physiological role of DES is similar with the natural estrogen, but DES is more stable and can remain in human body for a much longer time. Recently, the toxicological and carcinogenic properties of DES to animals and human beings have caused widespread concern. After being excessively exposed to DES, the male mice increase the risk of reproductive tract lesions and immunological dysfunctions [2-4]. Long-term intake of DES also raises the possibility of ovarian and breast cancers due to its genotoxicity [5-7]. However, DES can improve the fat deposition and quality of meat as a growth promotant $[8,9]$. And it is still commonly abused on growth and survival parameters or sex reversal procedure in mariculture engineering for pursuit of huge economic interests [10]. DES may exist in aquatic products or run into the

\footnotetext{
* Corresponding author.

E-mail address: lxchen@yic.ac.cn (L. Chen).
}

water by urine and feces, which has seriously threatened human health. Therefore, it is desirable to develop simple and direct methods for the detection of DES.

To date, several methods have been established to detect DES, such as high-performance liquid chromatography (HPLC), enzymelinked immunosorbent assay (ELISA), electrochemical techniques, and chemiluminescence [11-18]. HPLC can detect DES with good sensitivity and selectivity, but it usually needs sophisticated sample pretreatment $[11,12]$. ELISA is a quite sensitive approach, but preparation of antibody is time-consuming and troublesome [13-15]. The electrochemical techniques and chemiluminescence show low detection limit with good selectivity, but usually interfered by complex matrices [16-18]. Compared with these methods, fluorescence methods are outstanding due to its excellent sensitivity and selectivity. And colorimetric methods are much simpler and are able to be recognized by naked eyes. The two mentioned methods can be easily utilized in the on-site test $[19,20]$. However, to date, few colorimetric and fluorescence methods have been developed for detecting DES, especially the near-infrared (NIR) probes, which can avoid background fluorescence of dissolved organic compounds in natural seawater and bio-samples 
[21]. In this work, we developed a NIR probe Cy-DES to sensitively and selectively detect DES in both colorimetry and fluorescence method. The typical NIR dye heptamethine cyanine is chosen as the fluorophore, which has drawn great interest because of its high molar extinction coefficient and low cytotoxicity. In presence of DES, the self-aggregation of probe Cy-DES occurs, which leads to obvious changes in its spectroscopic properties including UV-Vis absorption spectra and fluorescence spectra. Take this advantage, the probe Cy-DES can successfully detect DES in complex matrices such as seawater, shrimp and fish samples. As a simple, sensitive, and nake-eye recognizable probe, Cy-DES may be conveniently utilized to detect DES on-site.

\section{Experimental}

\subsection{Chemicals and instruments}

All the chemicals used in the experiments were analytical reagent grade. UV-Vis spectra were measured on a $\mu$-Quant microplate reader Nanodrop 2000C (Thermo Scientific, USA) with a $1 \mathrm{~cm}$ quartz cell. Fluorescence spectra were quantitatively measured by FluoroMax-4 spectrofluorometer with a xenon lamp and $1 \mathrm{~cm}$ quartz cells. Cytotoxicity assay was carried out with a microplate reader (TECAN Infinite 200). High-resolution mass spectra were carried on LCQ Fleet LC-MS System (Thermo Fisher Scientific). ${ }^{1} \mathrm{H}$ NMR and ${ }^{13} \mathrm{C}$ NMR spectra were carried on a Bruker spectrometer.

\subsection{Preparation of the probe Cy-DES}

Ketone-Cy ( $100 \mathrm{mg}, 0.19 \mathrm{mmol})$ was synthesized in our laboratory [22] and triethylamine $(278 \mu \mathrm{L}, 0.6 \mathrm{mmol})$ was dissolved in $15 \mathrm{~mL}$ anhydrous $\mathrm{CH}_{2} \mathrm{Cl}_{2}$ at $0^{\circ} \mathrm{C}$. Then propionyl chloride $(16.5 \mathrm{~mL}$, $0.19 \mathrm{mmol}$ ) was added dropwise during $30 \mathrm{~min}$ (Scheme S1). The mixture was stirred $24 \mathrm{~h}$ at $25^{\circ} \mathrm{C}$. After evaporated in vacuo, the crude product was obtained as a deep green solid. Finally, the probe $\mathrm{Cy}$-DES was isolated by silica chromatography eluted with $\mathrm{CH}_{2} \mathrm{Cl}_{2} / \mathrm{CH}_{3} \mathrm{OH}(4: 1, \mathrm{v} / \mathrm{v}$ ) as a green solid ( $35 \mathrm{mg}$, yield $31 \%) .{ }^{1} \mathrm{H} N M R$ (500 MHz, DMSO) $\delta 8.28-8.25(\mathrm{~d}, 1 \mathrm{H}), 7.61-7.59(\mathrm{~m}, 2 \mathrm{H}), 7.44(\mathrm{~m}$, $1 \mathrm{H}), 7.27(\mathrm{~m}, 2 \mathrm{H}), 6.35-6.32(\mathrm{~d}, 1 \mathrm{H}), 6.25-6.22(\mathrm{~d}, 1 \mathrm{H}), 4.24-4.22(\mathrm{~s}$, $4 \mathrm{H}), 4.05-4.00(\mathrm{~m}, 2 \mathrm{H}), 2.91-2.89(\mathrm{~m}, 4 \mathrm{H}), 2.73-2.67(\mathrm{~d}, 2 \mathrm{H}), 1.99$ (s, 6H), 1.31-1.16 (m, 17H). ${ }^{13} \mathrm{C}$ NMR (126 MHz, DMSO) $\delta 203.18$, $195.65,179.12,174.43,172.06,171.51,170.78,158.94,153.23$, $142.13,141.54,139.63,129.11,125.47,122.97,121.62,111.67$, 100.91, 60.07, 49.04, 48.86, 27.71, 24.28, 21.19, 20.95, 14.60, 12.66, 9.64. LC-MS (ESI+): $m / z \mathrm{C}_{37} \mathrm{H}_{45} \mathrm{~N}_{2} \mathrm{O}_{2}{ }^{+}$calculated. 549.3476, found $\left[\mathrm{M}^{+}\right]$549.3473. Elemental analysis calculated (\%) for $\mathrm{C}_{37} \mathrm{H}_{45} \mathrm{~N}_{2} \mathrm{O}_{2}{ }^{+}$: C, 80.9; H, 8.2; N, 5.1 O, 5.8; found: C, 80.9; H, 8.3; N, 5.1; O, 5.7.

\subsection{Detection of DES by probe Cy-DES}

All absorption and fluorescence spectra were detected in 1.0$\mathrm{cm}$ cuvette cells. Before measurement, $1 \mu \mathrm{M}$ probe Cy-DES was incubated with different concentrations of DES in $50 \mathrm{mM}$ HEPES buffer at $37^{\circ} \mathrm{C}$ for $15 \mathrm{~min}$. The absorption spectra of Cy-DES were detected from 350 to $850 \mathrm{~nm}$. The fluorescence spectra of Cy-DES were detected from 740 to $880 \mathrm{~nm}$ with excitation at $720 \mathrm{~nm}$.

\subsection{Detection of DES in real samples}

Standard seawater samples were purchased from China Second Institute of Oceanography, State Oceanic Administration. Before UV-Vis detection, seawater samples were filtered through $0.22 \mu \mathrm{m}$ membrane to remove particulate matters. Shrimp and fish samples were pretreated according to the literature [23]. $10 \mathrm{~g}$ shrimp and fish samples were added to $100 \mathrm{~mL}$ containers. And $50 \mathrm{~mL}$ methanol was added to the containers. The samples were extracted by ultrasonic for $1 \mathrm{~h}$ after adequate shaking. And supernatants were collected by centrifuging at 10,000 rpm for $5 \mathrm{~min}$. After evaporation, the residue was dissolved in $1 \mathrm{~mL}$ of methanol and the solution was diluted to $50 \mathrm{~mL}$ with ultrapure water. Then the pre-treated seawater, shrimp and fish samples were spiked with different concentrations of DES and detected by our probe and high performance liquid chromatography (HPLC) method. The HPLC method for detection of DES was carried out with the mobile phase consisting acetonitrile-water $(70: 30, \mathrm{v} / \mathrm{v})$ at a flow rate $1.0 \mathrm{~mL} / \mathrm{min}$. The wavelength used to measure DES was set at $241 \mathrm{~nm}$ [12].

\section{Results and discussion}

\subsection{Probe design and proposed mechanism}

We designed and synthesized a colorimetric and near-infrared (NIR) fluorescent probe Cy-DES for the rapid and selective detection of DES (Scheme 1). The probe Cy-DES was developed by equipping heptamethine cyanine with propionyl chloride. After equipped with propionyl chloride, the probe's emission wavelength was centered at $790 \mathrm{~nm}$ (Fig. S1), because the heptamethine cyanine changed from ketone form to enol form and delocalized the positive charge by resonance over both nitrogen atoms (Schemes 1 and S1) $[24,25]$. This NIR probe Cy-DES was more optimal for detecting DES in seawater and bio-samples with its outstanding advantage of avoiding background fluorescence of other dissolved organic compounds. We anticipate that the conjugated system of DES delocalized the negative charge by acrossing both phenolic hydroxyl under alkaline environment [26]. The intermolecular aggregation occurs due to electrostatic interaction between cyanine dyes and DES, which leads to changes in the spectral properties of probe Cy-DES including the fluorescence quenching and the absorption hypsochromic shift $[27,28]$.

However, the absorption hypsochromic shift of cyanine dye can also be induced by the modulation of intramolecular polymethine $\pi$-electron system with the cleavage of the ester bond $[22,29]$ To verify the proposed mechanism of the probe Cy-DES for the detection of DES, more experiments were performed from different aspects. As shown in Fig. S2, the absorption spectrum of $\mathrm{Cy}$-DES did not change in the presence of $1 \mathrm{mM}$ phenol, which might cleave the ester bond of Cy-DES by its nucleophilic characteristic. The results indicated that the fluorescence/UV spectra changes of probe Cy-DES were not induced by phenolic hydroxyl. After the probe Cy-DES incubated with $10 \mu \mathrm{M}$ DES at $30^{\circ} \mathrm{C}$ for $20 \mathrm{~min}$, the emission peak of ketone-Cy did not emerge, which further verified that the ester bond of probe Cy-DES could not be cleaved by phenolic hydroxyl. As shown in Fig. S3, the intermolecular aggregation of Cy-DES in absence/presence of DES was clearly observed from the images collected by the optical microscopy [30]. Therefore, the detection mechanism of DES by probe Cy-DES was not caused by the modulation of intramolecular polymethine $\pi$-electron system but by the intermolecular aggregation. Considering that the structure of Cy-DES and DES are both in-plane charge-separation, the aggregation of Cy-DES may be similar to the H-aggregates of cyanine dye [31-33], which forms as a plane-to-plane stacking and is characterized by an obvious hypsochromically shifted absorption band (Scheme 1). To further investigate the fluorescence quenching mechanism, the fluorescence intensity quenching data was handled referred to Stern-Volmer (SV) equation:

$\frac{I_{0}}{I}=1+K_{\mathrm{sv}}[Q]$

where $I / I_{0}$ meant the fluorescence intensity of Cy-DES with/without $D E S$, respectively. And $K_{\text {sv }}$ meant the quenching constant. As shown in Fig. 1, the SV plots were nearly linear at low DES concentrations, 


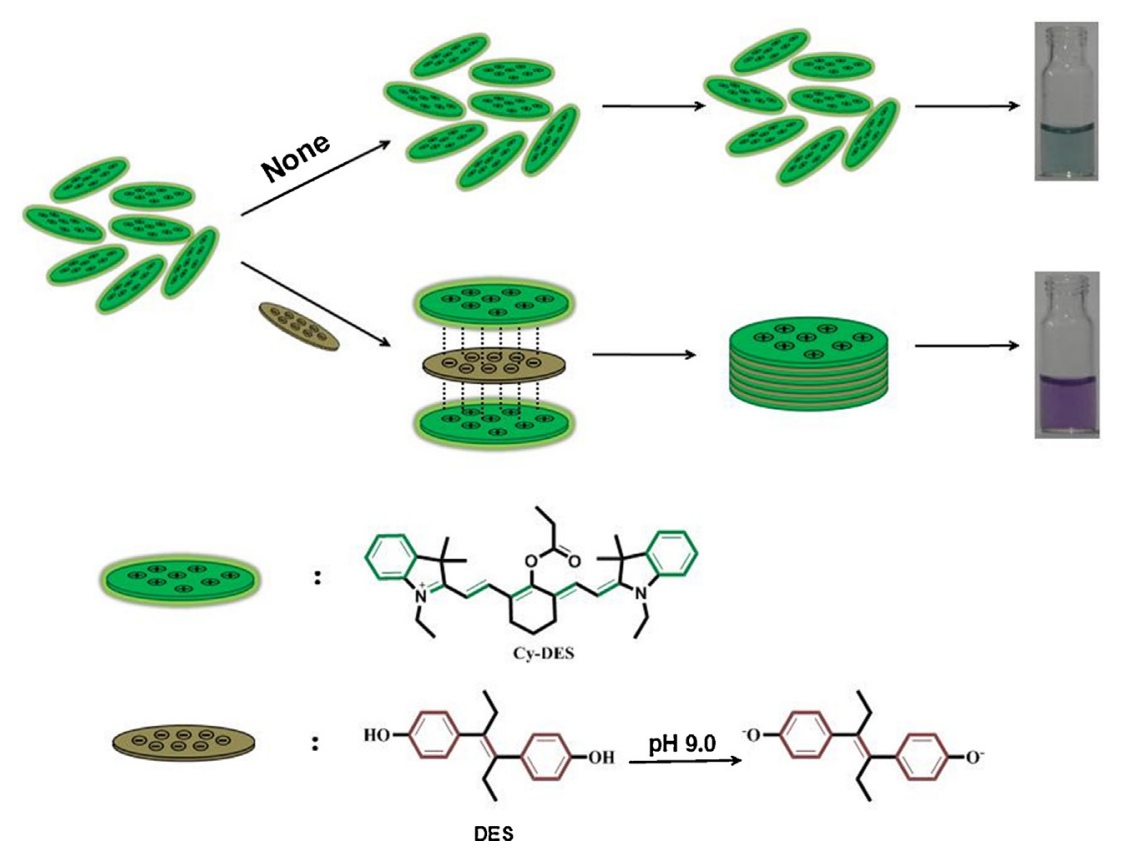

Scheme 1. Proposed mechanism of probe Cy-DES for the detection of DES.

which began to diverge from linearity and bended upwards with the concentration increasing. The results indicated a combination of dynamic and static quenching within the intermolecular aggregation of Cy-DES in presence of DES [34]. The quenching constant $K_{\text {sv }}=1.3 \times 10^{5}$ by the fitting of the SV plot, which demonstrated a high-quenching ability of DES to probe Cy-DES.

\subsection{The detection of DES by probe Cy-DES}

To test the viability of probe Cy-DES, the optimal condition was firstly investigated. As shown in Fig. S4, the best performance of probe $\mathrm{Cy}$-DES for the detection of DES was in $50 \mathrm{mM}$ B-R solutions, $\mathrm{pH} 9.0$ and incubated at $30^{\circ} \mathrm{C}$ for $20 \mathrm{~min}$. Nextly, probe Cy-DES

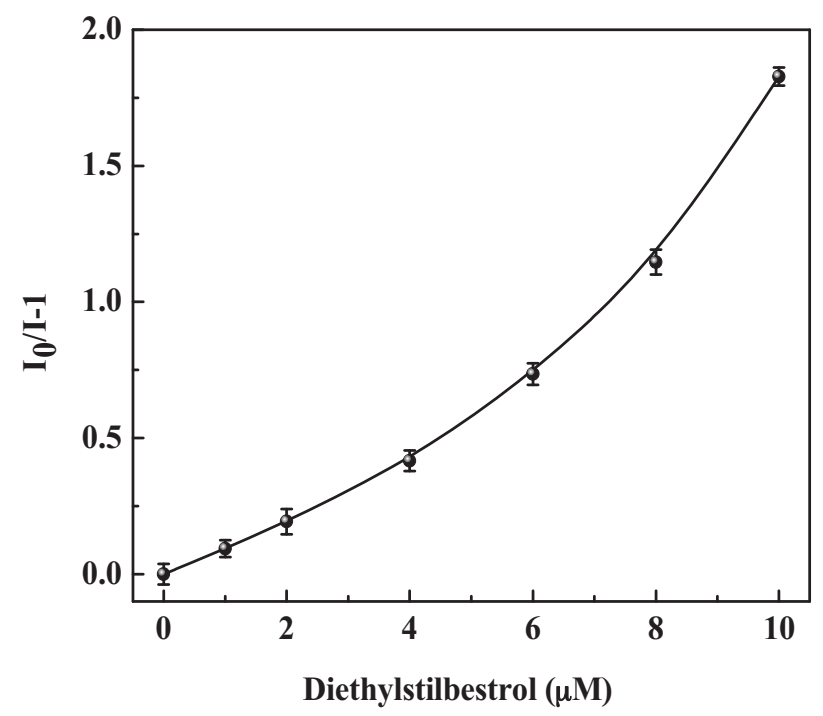

Fig. 1. The Stern-Volmer equation plot of Cy-DES in the presence of DES. Our probe Cy-DES $(1 \mu \mathrm{M})$ with different concentrations of DES $(0,1.0,2.0,4.0,6.0,8.0$ and $10 \mu \mathrm{M}$ ) in $50 \mathrm{mM} \mathrm{B}-\mathrm{R}$ solutions, $\mathrm{pH} 9.0$ and incubated at $30^{\circ} \mathrm{C}$ for $20 \mathrm{~min}$. Data were the means for three independent experiments. with various concentrations of DES $(0,1.0,2.0,4.0,6.0,8.0,10,15$ and $20 \mu \mathrm{M}$ ) was performed under this test condition. As shown in Fig. 2a, the UV-Vis absorption of probe Cy-DES was centered at $785 \mathrm{~nm}$, which displayed a green color. In the presence of different concentrations of DES, the absorption peak at $785 \mathrm{~nm}$ decreased gradually and a new absorption peak at $550 \mathrm{~nm}$ raised with a large hypsochromic-shift of $235 \mathrm{~nm}$, which indicated the intermolecular aggregation of Cy-DES. And the color of Cy-DES changed from green to purple, which can be clearly recognized by naked eyes. The probe Cy-DES showed strong fluorescence intensity at $790 \mathrm{~nm}$, a near infrared region. After DES added into the solution, a remarkable fluorescence intensity decrease was observed and the fluorescence quenching efficiency of probe Cy-DES increased gradually to $78 \%$, which indicated the strong electrostatic interactions between probe Cy-DES and DES after intermolecular aggregation (Fig. 2c). At pH 9.0, the DES was mainly in ionic form because of the dissociation of phenolic hydroxyl groups and the negative charge was delocalized in the conjugated system [35]. Therefore, the probe Cy-DES with positive charge was easily attracted by DES and intermolecular aggregation occurred, which induced obvious changes of Cy-DES spectral properties. As shown in Fig. 2b and d, both the UV-Vis absorption and the fluorescence intensity of Cy-DES were linearly proportional $(r=0.9997)$ to the DES concentrations from 1 to $8 \mu \mathrm{M}$ because the colorimetric signals accompanied with fluorescent signals were both generated by the intermolecular aggregation between Cy-DES and DES. And the detection limit (LOD) for DES was $0.2 \mu \mathrm{M}$ ( signal-to-noise $(S / N)$ ratio=3), which was similar with the HPLC method by solid-phase extraction for the analysis of diethylstilbestrol $(\mathrm{LOD}=0.22 \mu \mathrm{M})[23]$ and the ELISA method by metal ions-based immunosensor for determination of diethylstilbestrol $(\mathrm{LOD}=0.38 \mu \mathrm{M})[14]$. We also investigated the reversibility of probe Cy-DES in the detection of DES. The reversibility of the intermolecular aggregation of heptamethine cyanine usually depends on environmental parameters especially $\mathrm{pH}$ [27]. However, the intermolecular aggregation of probe DES in the presence of DES was not reversible by regulating $\mathrm{pH}$ values, which probably due to the strong intermolecular forces between probe Cy-DES and DES. Additionally, we estimated the cytotoxicity of Cy-NB by MTT assays with the help of HeLa, HepG2, and MCF-7 cells. As shown in Fig. S6, the 

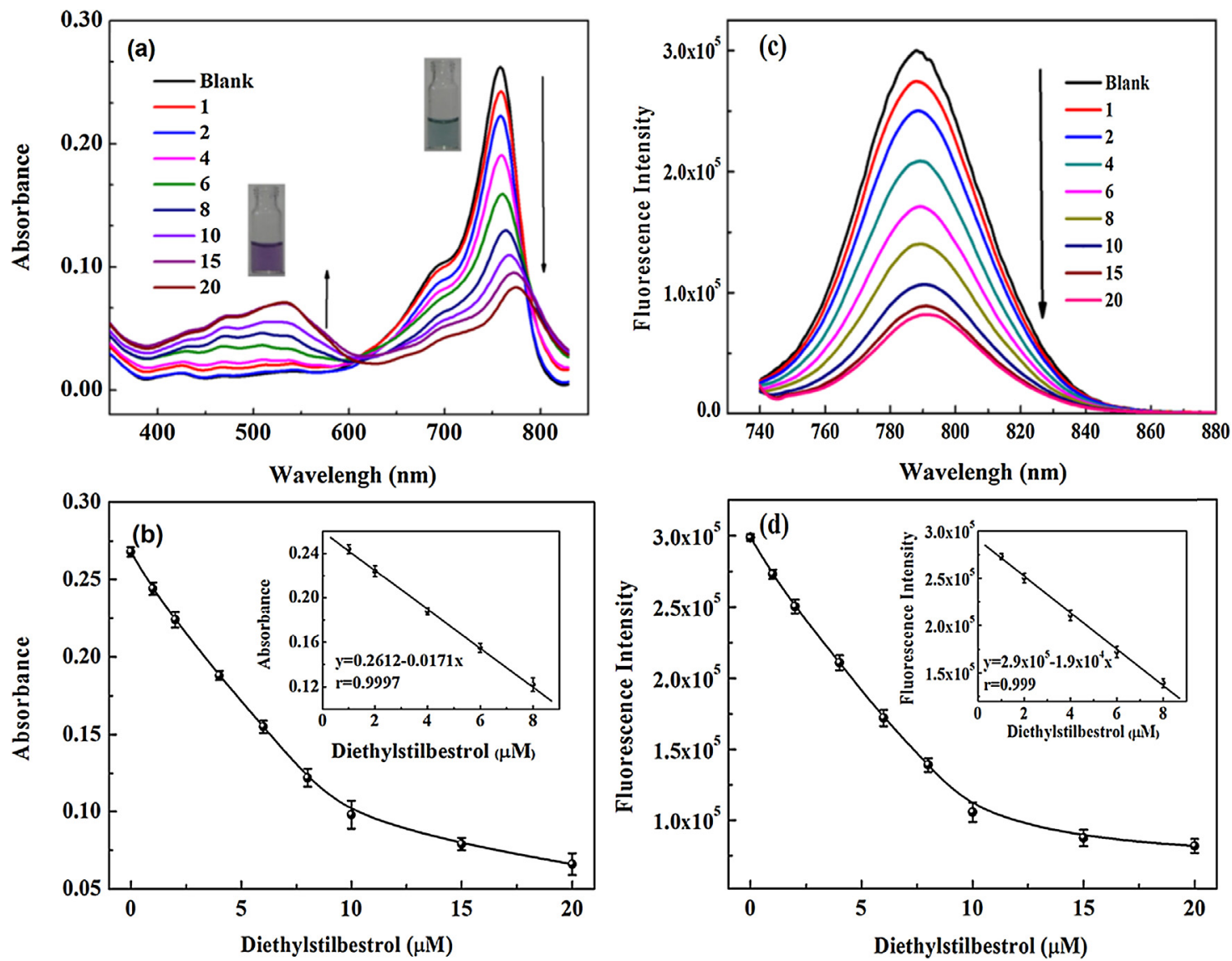

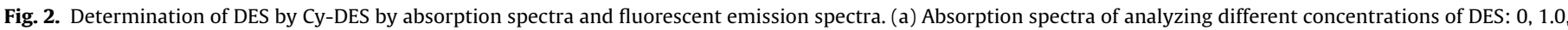

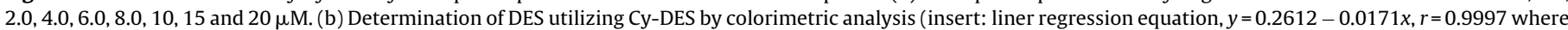

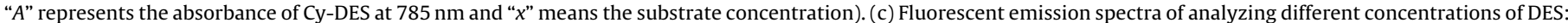

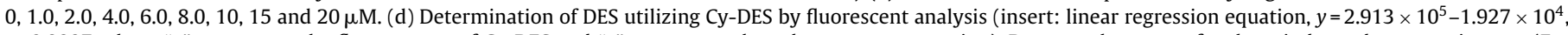

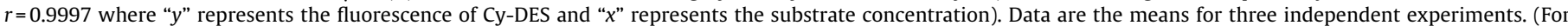
interpretation of the references to color in this figure legend, the reader is referred to the web version of this article.)

cellular viability was almost $99 \%$ in the presence of $1 \mu \mathrm{M}$ Cy-DES, which indicated the low cytotoxicity of probe Cy-DES. All above results indicated that our colorimetric and fluorescent probe $\mathrm{Cy}-$ DES can be utilized to detect DES with high sensitivity, which are expected to achieve the simple and sensitive detection of DES in real water and bio-samples.

\subsection{Selectivity of Cy-DES to DES}

To evaluate the selectivity of the probe Cy-DES toward DES, the fluorescence and absorption spectra response of the DES as well as possible interferences including estrogens, metal ions and fish drugs were taken into consideration. To examine the interference of other estrogens whose structure were quite similar with DES, the probe Cy-DES was incubated with $10 \mu \mathrm{M}$ DES, $10 \mu \mathrm{M}$ bisphenol A, $100 \mu \mathrm{M}$ estrone, $17-\alpha$-estradiol, $\beta$-estradiol and estriol, respectively. As shown in Fig. 3a, our probe Cy-DES showed a good selectivity toward DES over these estrogens. Fish drugs are important interferences in the detection of DES as they are commonly used for disease management in mariculture engineering. To investigate the selectivity of Cy-DES to DES over fish drugs, the probe Cy-DES was incubated with $10 \mu \mathrm{M}$ DES, $100 \mu \mathrm{M}$ furazolidone, sulfapyridine, sulfadimidine, sulfadoxine, trichlorphon, sulfathiazole, bromophos methyl, bromophos ethyl and diazinon, respectively. As shown in Fig. 3b, our probe Cy-DES showed an excellent selectivity toward DES over these fish drugs. The detection of DES concentration in aquaculture seawater was an important mission in the design of probe Cy-DES. To achieve this aim, the detection of DES by probe Cy-DES should not be influenced by high salinity. Therefore, we examined the influence of metal ions on the detection of DES by probe Cy-DES. The probe Cy-DES was incubated with $10 \mu \mathrm{M}$ DES in $50 \mathrm{mM} \mathrm{B}-\mathrm{R}$ solutions and $10 \mu \mathrm{M}$ DES with $0.5 \mathrm{M} \mathrm{Na}^{+}, 100 \mu \mathrm{M}$ $\mathrm{K}^{+}, \mathrm{Li}^{+}, \mathrm{Ca}^{2+}, \mathrm{Cd}^{2+}, \mathrm{Mg}^{2+}, \mathrm{Pb}^{2+}, \mathrm{Cu}^{2+}, \mathrm{Zn}^{2+}, \mathrm{Hg}^{2+}, \mathrm{Mn}^{2+}$, respectively. As shown in Fig. 3c, the detection of DES by probe Cy-DES would not be influenced by these metal ions. Additionally, we also verified the selectivity of probe Cy-DES to DES on 96-well microtiter plates. As shown in Fig. 3d, DES could be easily picked out from other potential interferences in presence of probe Cy-DES. All above results indicated that our probe Cy-DES owned an excellent selectivity toward DES over other interferences.

\subsection{Detection of DES in real samples}

To study the matrix effect on the detection of DES by probe Cy-DES, seawater, shrimp and fish samples spiked with 2.0, 4.0, 8.0 $\mu \mathrm{M}$ DES were tested. The seawater samples were first filtered to remove insoluble substances. However, the dissolved organic compounds including amino acids, sugar acids, carboxylic acids and fatty acids were still existed in the natural seawater, whose fluorescence emission spectra were usually located from 300 to $400 \mathrm{~nm}$ [21]. Therefore, the fluorescence probes with a shorter emission wavelength are easy to be influenced by these dissolved organic 


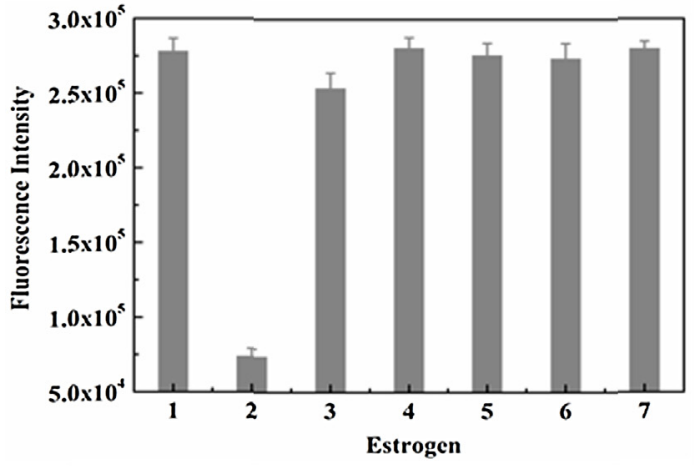

(a)

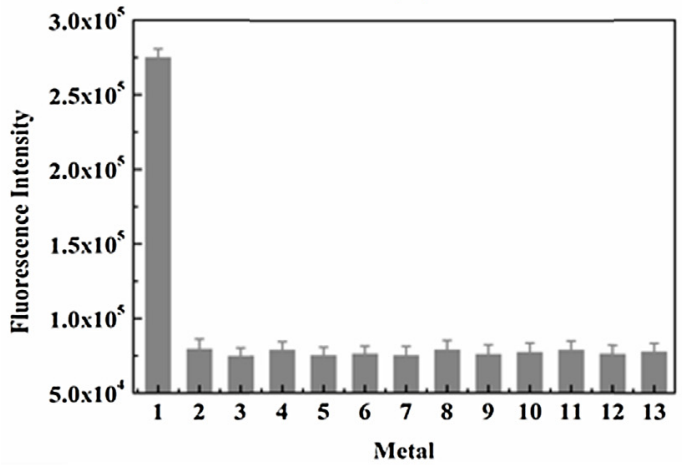

(c)

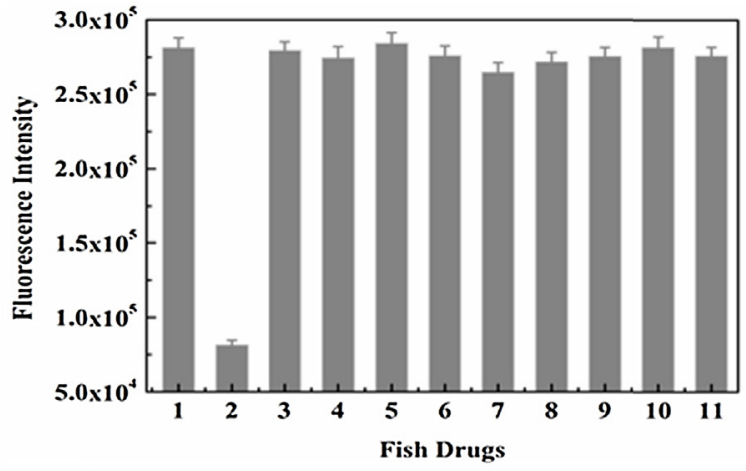

(b)

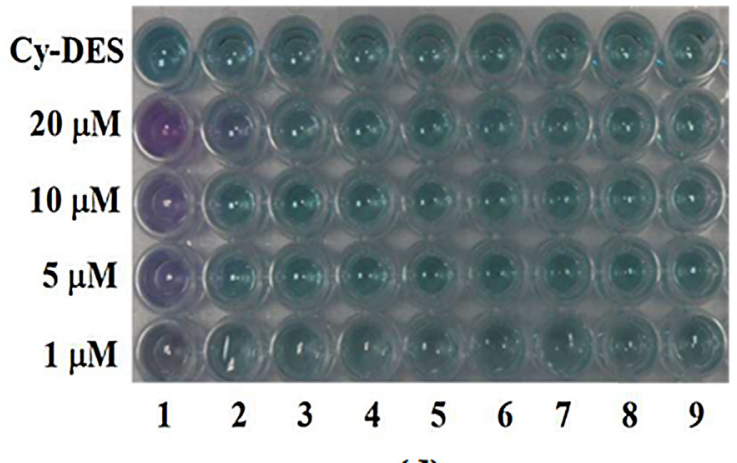

(d)

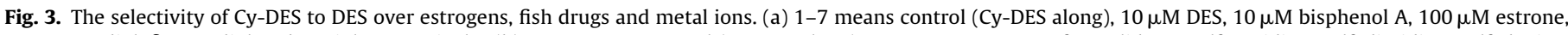

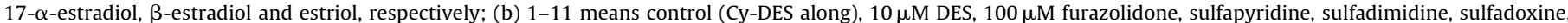

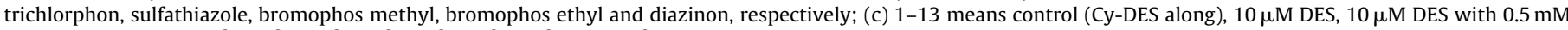

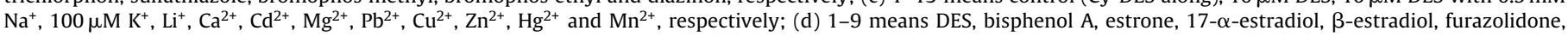
sulfapyridine, sulfathiazole, and diazinon. Data are the means for three independent experiments.

Table 1

The results obtained by Cy-DES and HPLC for the detection of DES.

\begin{tabular}{|c|c|c|c|c|c|}
\hline Sample & Spiked $(\mu \mathrm{M})$ & This method $(\mu \mathrm{M})$ & Recovery (\%) & $\operatorname{HPLC}(\mu \mathrm{M})$ & Recovery (\%) \\
\hline \multirow[t]{4}{*}{ Seawater } & 0.0 & ND & ND & ND & ND \\
\hline & 2.0 & $1.96 \pm 0.14$ & $98.0 \pm 7.0$ & $2.08 \pm 0.09$ & $104.0 \pm 4.5$ \\
\hline & 4.0 & $3.68 \pm 0.27$ & $92.0 \pm 6.8$ & $3.87 \pm 0.21$ & $96.8 \pm 5.2$ \\
\hline & 8.0 & $8.16 \pm 0.51$ & $102.0 \pm 6.4$ & $7.85 \pm 0.37$ & $98.1 \pm 4.6$ \\
\hline \multirow[t]{4}{*}{ Shrimp } & 0.0 & ND & ND & ND & ND \\
\hline & 2.0 & $1.86 \pm 0.21$ & $93.0 \pm 10.5$ & $1.97 \pm 0.13$ & $98.5 \pm 6.5$ \\
\hline & 4.0 & $4.17 \pm 0.28$ & $104.2 \pm 7.0$ & $4.21 \pm 0.19$ & $105.2 \pm 4.7$ \\
\hline & 8.0 & $7.69 \pm 0.63$ & $96.1 \pm 7.9$ & $8.19 \pm 0.57$ & $102.4 \pm 7.1$ \\
\hline \multirow[t]{4}{*}{ Fish } & 0.0 & ND & ND & ND & ND \\
\hline & 2.0 & $2.13 \pm 0.09$ & $106.5 \pm 4.5$ & $1.89 \pm 0.07$ & $94.5 \pm 3.5$ \\
\hline & 4.0 & $4.19 \pm 0.36$ & $104.7 \pm 9.0$ & $3.89 \pm 0.31$ & $97.2 \pm 7.7$ \\
\hline & 8.0 & $8.28 \pm 0.46$ & $103.4 \pm 5.8$ & $7.89 \pm 0.57$ & $98.6 \pm 7.1$ \\
\hline
\end{tabular}

ND means not detectable.

compounds. As shown in Table 1, the detected concentrations of DES by probe Cy-DES were $1.96,3.68$ and $8.16 \mu \mathrm{M}$, respectively, which were approximate to the values of $2.08,3.87$ and $7.85 \mu \mathrm{M}$ detected by HPLC, respectively. The results of the present colorimetric and fluorescent probe Cy-DES were in excellent agreement with those obtained by HPLC, which indicated that the probe CyDES was suitable for the direct detection of DES concentration in seawater samples. And the detection of DES by Cy-DES would not be influenced by dissolved organic compounds in seawater, which benefited from its NIR property. In the shrimp and fish samples, the DES in these samples could also be successfully detected by Cy-DES and the results agreed well with those obtained by the traditional method HPLC (Table 1), which indicated that the residual DES in aquatic products could be well detected by the developed probe Cy-DES. All these results verified that our probe Cy-DES was credible and practically feasible for detecting DES in real samples.

\section{Conclusions}

In summary, we have developed a new and simple colorimetric and fluorescent probe Cy-DES for the selectively detection of DES. The probe is established based on the intermolecular aggregates between Cy-DES and DES by strong electrostatic interaction. In the presence of DES, an obvious absorption hypsochromic-shift of Cy-DES occurs and the color of the solution changes from blue to purple. As a result, the probe can conveniently detect DES by the naked eyes. The detection of DES by probe Cy-DES cannot be 
influenced by high salinity. As a kind of NIR probe, the probe CyDES can effectively avoid the background fluorescence of dissolved organic compounds in natural seawater. The probe Cy-DES has been successfully utilized to detect DES in complicated matrix such as seawater, shrimp and fish samples. In short, our probe Cy-DES owns great advantages and shows potential application in the DES detection in real samples.

\section{Acknowledgments}

We thank the National Science Foundation of China (21575159, 21405172, 21275158, and 31370108), the Strategic Priority Research Program of the Chinese Academy of Sciences (XDA11020405), the Key Research Program of the Chinese Academy of Sciences (KZZD-EW-14), the program of Youth Innovation Promotion Association, CAS (2015170), and One Hundred-Talent Plan of Chinese Academy of Sciences.

\section{Appendix A. Supplementary data}

Supplementary data associated with this article can be found, in the online version, at http://dx.doi.org/10.1016/j.snb.2015.10.014.

\section{References}

[1] O.W. Smith, Diethylstilbestrol in the prevention and treatment of complications of pregnancy, Obstet. Gynecol. Surv. 4 (1949) 190-191.

[2] J.A. McLachlan, R.R. Newbold, B. Bullock, Reproductive tract lesions in male mice exposed prenatally to diethylstilbestrol, Science 190 (1975) 991-992.

[3] M.I. Luster, R.E. Faith, J.A. McLachlan, G.C. Clark, Effect of in utero exposure to diethylstilbestrol on the immune response in mice, Toxicol. Appl. Pharm. 47 (1979) 279-285.

[4] P.H. Bick, A.N. Tucker, K.L. White Jr., M.P. Holsapple, Effects of subchronic exposure to diethylstilbestrol on humoral immune function in adult female (C3B6)F1 mice, Immunopharmacology 7 (1984) 27-39.

[5] E.R. Greenberg, A.B. Barnes, L. Resseguie, J.A. Barrett, S. Burnside, L.L. Lanza, R. Neff, M. Stevens, R. Young, T. Colton, Breast cancer in mothers given diethylstilbestrol in pregnancy, N. Engl. J. Med. 311 (1984) 1393-1398.

[6] A. Gladek, J.G. Liehr, Mechanism of genotoxicity of diethylstilbestrol in vivo, J. Biol. Chem. 264 (1989) 16847-16852.

[7] J. Blatt, L. Van Le, T. Weiner, S. Sailer, Ovarian carcinoma in an adolescent with transgenerational exposure to diethylstilbestrol, J. Pediat. Hematol. Oncol. 25 (2003) 635-636.

[8] F.W. Lorenz, The influence of diethylstilbestrol on fat deposition and meat quality in chickens, Poult. Sci. 24 (1945) 128-134.

[9] K.E. McMartin, K.A. Kennedy, P. Greenspan, S.N. Alam, P. Greiner, J. Yam, Diethylstilbestrol: a review of its toxicity and use as a growth promotant in food-producing animals, J. Environ. Pathol. Toxicol. 1 (1978) 279.

[10] J. Król, W. Pobłocki, T. Bockenheimer, P. Hliwa, Effect of diethylstilbestrol (DES) and $17 \beta$-estradiol (E2) on growth, survival and histological structure of the internal organs in juvenile European catfish Silurus glanis (L.), Aquac. Int. 22 (2014) 53-62.

[11] A.R. Williams, S.A. Winfield, R.C. Belloli, Rapid, specific method for diethylstilbestrol analysis using an in-line photochemicals reactor with high-performance liquid chromatography and fluorescence detection, J. Chromatogr. A 235 (1982) 461-470.

[12] Y. Yang, J. Chen, Y.P. Shi, Determination of diethylstilbestrol in milk using carbon nanotube-reinforced hollow fiber solid-phase microextraction combined with high-performance liquid chromatography, Talanta 97 (2012) 222-228.

[13] G. Degand, P. Schmitz, G. Maghuin-Rogister, Enzyme immunoassay screening procedure for the synthetic anabolic estrogens and androgens diethylstilbestrol, nortestosterone, methyltestosterone and trenbolone in bovine urine, J. Chromatogr. B 489 (1989) 235-243.

[14] S. Zhang, B. Du, H. Li, X. Xin, H. Ma, D. Wu, L. Yan, Q. Wei, Metal ions-based immunosensor for simultaneous determination of estradiol and diethylstilbestrol, Biosens. Bioelectron. 52 (2014) 225-231.

[15] S. Liu, Q. Lin, X. Zhang, X. He, X. Xing, W. Lian, J. Li, M. Cui, J. Huang, Electrochemical immunosensor based on mesoporous nanocomposites and HRP-functionalized nanoparticles bioconjugates for sensitivity enhanced detection of diethylstilbestrol, Sens. Actuators B Chem. 166 (2012) 562-568

[16] D. Lu, S. Lin, L. Wang, X. Shi, C. Wang, Y. Zhang, Synthesis of cyclodextrin-reduced graphene oxide hybrid nanosheets for sensitivity enhanced electrochemical determination of diethylstilbestrol, Electrochim. Acta 85 (2012) 131-138.
[17] S. Zhang, K. Wu, S. Hu, Voltammetric determination of diethylstilbestrol at carbon paste electrode using cetylpyridine bromide as medium, Talanta 58 (2002) 747-754

[18] Q.L. Zhang, J. Li, T.T. Ma, Z.T. Zhang, Chemiluminescence screening assay for diethylstilbestrol in meat, Food Chem. 111 (2008) 498-502.

[19] K. Yin, B. Li, X. Wang, W. Zhang, L. Chen, Ultrasensitive colorimetric detection of $\mathrm{Cu} 2+$ ion based on catalytic oxidation of L-cysteine, Biosens. Bioelectron. 64 (2015) 81-87.

[20] Z.H. Liu, S. Devaraj, C.R. Yang, Y.P. Yen, A new selective chromogenic and fluorogenic sensor for citrate ion, Sens. Actuators B Chem. 174 (2012) $555-562$.

[21] P.G. Coble, S.A. Green, N.V. Blough, R.B. Gagosian, Characterization of dissolved organic matter in the Black Sea by fluorescence spectroscopy, Nature 348 (1990) 432-435.

[22] K. Yin, F. Yu, W. Zhang, L. Chen, A near-infrared ratiometric fluorescent probe for cysteine detection over glutathione indicating mitochondrial oxidative stress in vivo, Biosens. Bioelectron. 74 (2015) 156-164.

[23] X. Jiang, C. Zhao, N. Jiang, H. Zhang, M. Liu, Selective solid-phase extraction using molecular imprinted polymer for the analysis of diethylstilbestrol, Food Chem. 108 (2008) 1061-1067.

[24] F. Yu, M. Gao, M. Li, L. Chen, A dual response near-infrared fluorescent probe for hydrogen polysulfides and superoxide anion detection in cells and in vivo, Biomaterials 63 (2015) 93-101.

[25] B.A. Armitage, DNA Binders and Related Subjects, vol. 253, Springer, Berlin; Heidelberg, 2005, pp. 55-76.

[26] E.J. Yearley, E.A. Zhurova, V.V. Zhurov, A.A. Pinkerton, Experimental electron density studies of non-steroidal synthetic estrogens: diethylstilbestrol and dienestrol, J. Mol. Struct. 890 (2008) 240-248.

[27] A. Mishra, R.K. Behera, P.K. Behera, B.K. Mishra, G.B. Behera, Cyanines during the 1990s: a review, Chem. Rev. 100 (2000) 1973-2012.

[28] H. Zheng, M. Yan, X.X. Fan, D. Sun, S.Y. Yang, L.J. Yang, J.D. Li, Y.B. Jiang, A heptamethine cyanine-based colorimetric and ratiometric fluorescent chemosensor for the selective detection of $\mathrm{Ag}^{+}$in an aqueous medium, Chem. Commun. 48 (2012) 2243-2245.

[29] Z. Guo, S. Nam, S. Park, J. Yoon, A highly selective ratiometric near-infrared fluorescent cyanine sensor for cysteine with remarkable shift and its application in bioimaging, Chem. Sci. 3 (2012) 2760-2765.

[30] E.S. Emerson, M.A. Conlin, A.E. Rosenoff, K.S. Norland, H. Rodriguez, D. Chin, G.R. Bird, The geometrical structure and absorption spectrum of a cyanine dye aggregate, J. Phys. Chem. C 71 (1967) 2396-2403.

[31] U. Rösch, S. Yao, R. Wortmann, F. Würthner, Fluorescent H-aggregates of merocyanine dyes, Angew. Chem. Int. Ed. 118 (2006) 7184-7188.

[32] T.E. Kaiser, H. Wang, V. Stepanenko, F. Würthner, Supramolecular construction of fluorescent J-aggregates based on hydrogen-bonded perylene dyes, Angew. Chem. Int. Ed. 46 (2007) 5541-5544.

[33] V. Kumar, G.A. Baker, S. Pandey, Ionic liquid-controlled J-versus H-aggregation of cyanine dyes, Chem. Commun. 47 (2011) 4730-4732.

[34] C.B. Murphy, Y. Zhang, T. Troxler, V. Ferry, J.J. Martin, W.E. Jones, Probing Förster and Dexter energy-transfer mechanisms in fluorescent conjugated polymer chemosensors, J. Phys. Chem. B 108 (2004) 1537-1543.

[35] M. Mei, J. Yu, X. Huang, H. Li, L. Lin, D. Yuan, Monitoring of selected estrogen mimics in complicated samples using polymeric ionic liquid-based multiple monolithic fiber solid-phase microextraction combined with high-performance liquid chromatography, J. Chromatogr. A 1385 (2015) 12-19.

\section{Biographies}

Kun Yin received his BS degree in aquaculture from China Agriculture University. He is currently a PhD student at Yantai Institute of Coastal Zone Research, Chinese Academy of Sciences. His scientific interest includes bioremediation and biosensor for environmental pollutants.

Fabiao Yu received his MS from Shandong Normal University in 2009. He then received his PhD degree from the Dalian University of Technology and Dalian Institute of Chemical Physics, Chinese Academy of Sciences, in 2013. He is currently an associate professor at the Yantai Institute of Coastal Zone Research, Chinese Academy of Sciences. His research interests focus on functional probe molecules, mainly in fluorescence and phosphorescence analysis.

Dongyan Liu received her PhD in Ocean University of China, in 2004, and has nearly 20 years' experience in marine ecological field. Now she is a professor in Yantai Institute of Coastal Zone Research, Chinese Academy of Sciences. She has published 143 research articles on coastal ecosystem, including over 40 international publications. Her current main research interest focusing on coastal marine ecosystem regime shift in response to an intensive human activity and a regional climate change in Bohai and Yellow Seas, China.

Zhihong Xie received her PhD in China Agriculture University in 2005, and has nearly 20 years' experience in environmental microbiology field. Now she is a professor in Yantai Institute of Coastal Zone Research, Chinese Academy of Sciences. She has published more than 40 research articles on environmental microbiology, including over 20 international publications. Her current main research interest 
focusing on microbial physiology and biochemistry and restoration of coastal wetlands by environmental microbiology.

Lingxin Chen received his $\mathrm{PhD}$ in analytical chemistry from Dalian Institute of Chemical Physics, Chinese Academy of Sciences, China, in 2003. After 2 years postdoctoral experience in the Department of Chemistry, Tsinghua University, China, he joined first as a BK21 researcher and then as a research professor in the Department of Applied Chemistry, Hanyang University, Ansan, Korea, in 2006. Now he is a professor in Yantai Institute of Coastal Zone Research, Chinese Academy of Sciences. His current research interests include chromatographic separation techniques and optical sensor technologies for environmental analysis using novel properties of materials such as functionalized nanoparticles and molecularly imprinted polymers. 